\title{
Contributing Ad Hoc Reviewers 2016
}

Rehabilitation Psychology publishes articles that capture the breadth of science and practice in rehabilitation psychology. To achieve this mission we rely on a diverse group of individuals who serve as ad hoc reviewers. We are grateful to the following individuals for their generous commitment of time and effort for the 2016 volume of Rehabilitation Psychology. If you would like to join this group of individuals by serving as an ad hoc reviewer please contact the editor at swegener@jhmi.edu.

Amy Allen

Kevin N. Alschuler

Herb Ames

Derek Ryan Anderson

Juan Alfonso Andrade Ortega

Erin Andrews

Patricia Arenth

Trey William Armstrong

Martha E. Banks

Sandra Bassett

Harald Baumeister

Kathleen T. Bechtold

Meghan Beier

Heather Belanger

Jacob A. Bentley

Jack W. Berry

Oscar Bienvenue

Malachy Bishop

Chris Blanchard

Arthur Blaser

Kathleen Bogart

Charles H. Bombardier

George A. Bonanno

Lisa Brenner

Patrick Brice

Jo Ann Brockway

Susanne M. Bruyere

Susan P Buckelew

Maggi A. Budd

Catherine Butz

Sandra Byers

Yue Cao

Bruce Caplan

Renan Castillo

Denise Catalano

Chetwyn Chan

Fong Chan

Jacob Yuichung Chan

Yuying Chen

Chung-Yi Chiu

Julie A. Chronister

Brigit K. Clancy

Wendy A. Coduti

Laura Coffey

John Connolly

Patrick Corrigan
Linda Denehy

Terri A. deRoon-Cassini

Deirdre Desmond

Gerald M. Devins

Nicole Ditchman

Jacobus Donders

Shengli Dong

Diana S. Dorstyn

Laura Dreer

Dana S. Dunn

Dawn M. Ehde

Daniel Eisenberg

Timothy R. Elliott

Doug Elliott

Rosalind Elliott

Ruth S. Farber

Pamela Fitzpatrick

Becca Floyd

Robert T. Fraser

Pamela Gallagher

Eric Garshick

Maryam Gholami

Robert L. Glueckauf

Lance Goetz

Yelena Goldin

Fernando Gonzalez

Christopher D. Graham

Anna E. Greer

Teresa Hagan

Kimberley Haines

Nan Zhang Hampton

H'Sien Hayward

Allen W. Heinemann

Debra Homa

Ramona Hopkins

Megan Hosey

Sigmund Hough

Matt Howard

Warren T. Jackson

Doug Johnson-Greene

Marie Johnston

Salene M. W. Jones

Gabrielle Jones
Shannon B. Juengst

Claire Z. Kalpakjian

Stuart Brian Kamenetsky

Kathy Kapp Simon

Robert Karol

Mary Kennedy

Thomas R. Kerkhoff

Elizabeth Kilgour

Jeong Han Kim

Sonya Kim

Kristine Kingsley

Daniel Klyce

Anna Kratz

James S. Krause

Margaret Legarreta

Hanoch Livneh

Sara Marie Locatelli

John Lucas

John McClure

Scott McDonald

Ashlee Brooke McKeon

Swati Mehta

Ivan Molton

Kimberley R. Monden

Dennis Moore

Donna Morere

Chelsea Morse

George Mugoya

Michelle Reyna

Nario-Redmond

Janet P. Niemeier

Barry P. Nierenberg

Karen Nieves-Lugo

Margaret Nosek

Rhoda Olkin

Tisha Joy Ornstein

Francesco Pagnini

Kenneth I. Pakenham

Paul B. Perrin

Carisa Perry-Parrish

Claudio Peter

Carrie Pilarski
Robert Pollard

Marcel Post

Karen Postal

Joseph F. Rath

Jennifer Reesman

J. Scott Richards

Diego Rivera

Cynthia Salorio

Jennifer Sánchez

Alecia Santuzzi

Eric Schmidt

Ron T. Seel

Mark Sherer

Arielle Silverman

Richard L. Skolasky

Keith J. Slifer

McKay Sohlberg

Robert Spencer

William Stiers

Kirk J. Stucky

Sarah Tlustos

Pegah Touradji

Aaron P Turner

Jay M. Uomoto

David E. Vance

Rodney D. Vanderploeg

Jesse Verschuren

Noa Vilchinsky

Shari Wade

Dorothy Wade

Dorothy Wade

Cynthia M. Ward

Seth Warschausky

Sandra Weintraub

Rhonda Williams

Amanda E. Young

Andrew Zabel

Lisa Zheng 